\title{
Diseño de espacios educativos significativos para el desarrollo de competencias en la infancia ${ }^{1}$ Designing meaningful educational spaces for the development
of competencies in childhood
}

\author{
Yenny Otálora Sevilla \\ Universidad del Valle, Colombia \\ yotalora@gmail.com
}

Artículo de reflexión recibido el 15/04/10 y aprobado el 31/05/10

\begin{abstract}
Resumen
Un espacio educativo significativo es un ambiente de aprendizaje que promueve y fortalece el desarrollo de competencias sociales y cognitivas en los niños. Este artículo ofrece elementos conceptuales y metodológicos de la psicología educativa que facilitan el diseńo de espacios educativos significativos para el desarrollo de competencias infantiles dentro y fuera del aula. Por una parte, se brinda una definición de ambiente de aprendizaje que permite comprenderlo como un espacio dinámico y complejo de construcción de conocimiento. De otra parte, teniendo en cuenta algunas consideraciones sobre la concepción de desarrollo infantil, se establecen cinco criterios para caracterizar los ambientes de aprendizaje como espacios educativos significativos: situaciones estructuradas, intensivas, extensivas, generativas y ricas en formas de interacción. Cada uno de los criterios se ilustra con ambientes de aprendizaje diseñados por agentes educativos de Colombia, basados en el uso de prácticas culturales de sus comunidades de origen. Las prácticas culturales resultaron ser pertinentes para el desarrollo de las competencias de sus nińos.
\end{abstract}

Palabras clave: Desarrollo infantil, Educación inicial, Ambiente de aprendizaje, Prácticas culturales

\begin{abstract}
A meaningful educational space is a learning environment that promotes and strengthens the development of social and cognitive competencies in children. This article offers conceptual and methodological elements from Educational Psychology that facilitates the design of significant educational spaces for the development of child competencies, both in and out of school. On the one hand, the learning environment is defined as a dynamic and complex space of construction of knowledge. On the other it takes into account some considerations about child development which help set five criteria to characterize learning environments as meaningful educational spaces: They are structured, intensive, extensive, generative and interactive situations. Each of these criteria is illustrated through learning environments designed by educators in Colombia, based on the use of cultural practices inherent their communities of origin. Cultural practices were found to be relevant to the development of their children's competencies.
\end{abstract}

Key words: Child development, early education, learning environment, cultural practices

1 Este artículo es producto de la investigación "Prueba piloto a escala real para la evaluación del aprendizaje de la escritura de numerales en comunidades escolares", código 110645221365, financiado por Colciencias, contrato 637-2008, presentado a través del Centro de Investigaciones en Psicología, Cognición y Cultura de la Universidad del Valle. 



\section{Introduccion}

La calidad del desarrollo cognitivo, social y afectivo de los niños depende en gran medida de la calidad de las prácticas en las que ellos participan. En esta medida, generar experiencias enriquecedoras que fortalezcan el desarrollo integral de los niños durante la primera infancia es un propósito que preocupa a instituciones educativas, agentes educativos y comunitarios, psicólogos y otros profesionales que están dedicados a su cuidado en el período de cero a seis años. Los espacios educativos constituyen ambientes de aprendizaje creados por estas personas con el objetivo de promover su crecimiento, su conocimiento del mundo y su actuación exitosa en él. Lograr que las prácticas y actividades propuestas en los contextos educativos lleguen a ser significativos para tales propósitos es una tarea que exige el establecimiento y uso de criterios que faciliten su diseńo y su implementación.

Este artículo brinda, desde la perspectiva de la psicología educativa, algunos elementos conceptuales y metodológicos sobre el diseño o enriquecimiento de ambientes de aprendizaje que pueden ser utilizados como espacios educativos que resulten significativos para el desarrollo de las competencias de los niños durante los primeros seis años de vida. El artículo presenta, inicialmente, una definición de ambiente aprendizaje y señala un conjunto de consideraciones sobre la concepción de desarrollo infantil a partir de las cuales se derivan implicaciones para la definición de un espacio educativo significativo. Se parte del supuesto que la concepción del sujeto que aprende y se desarrolla dirige el tipo de prácticas que se le proponen para su educación. Luego se propone ilustrar algunos criterios específicos para el diseño o enriquecimiento de espacios educativos significativos. A través de la revisión de éstos criterios el artículo promueve especialmente el uso de prácticas cotidianas y culturales de las comunidades a las que pertenecen los niños como espacios educativos que pueden llegar a ser pertinentes para el desarrollo de competencias en dominios específicos de conocimiento y a su vez, la construcción de la personalidad, la identidad cultural y, el sentido de pertenencia a la comunidad.

Con esta estrategia se busca contribuir al mejoramiento de la calidad de la educación inicial en contextos educativos formales y no formales, tanto urbanos como rurales, así como en comunidades culturales específicas.

\section{La definición de ambiente de aprendizaje}

Un ambiente de aprendizaje constituye un escenario de construcción de conocimiento en el que un agente educativo -o institución educativa, organización o grupo cultural-genera intencionalmente un conjunto de actividades y acciones 
dirigidas a garantizar la consecución de un objetivo de aprendizaje amplio que es pertinente para el desarrollo de competencias en uno o varios dominios de conocimiento de uno o más educandos pertenecientes a una cultura. El concepto de ambiente de aprendizaje no sólo se refiere a la totalidad de las actividades que giran alrededor de un objetivo de aprendizaje centrado en un conocimiento específico, un contenido temático o una habilidad, como tradicionalmente se concibe. Un ambiente de aprendizaje es un espacio estructurado en el que se articulan diversos elementos y relaciones necesarios para alcanzar tal objetivo. La figura 1 pretende ilustrar algunos de estos elementos y relaciones.

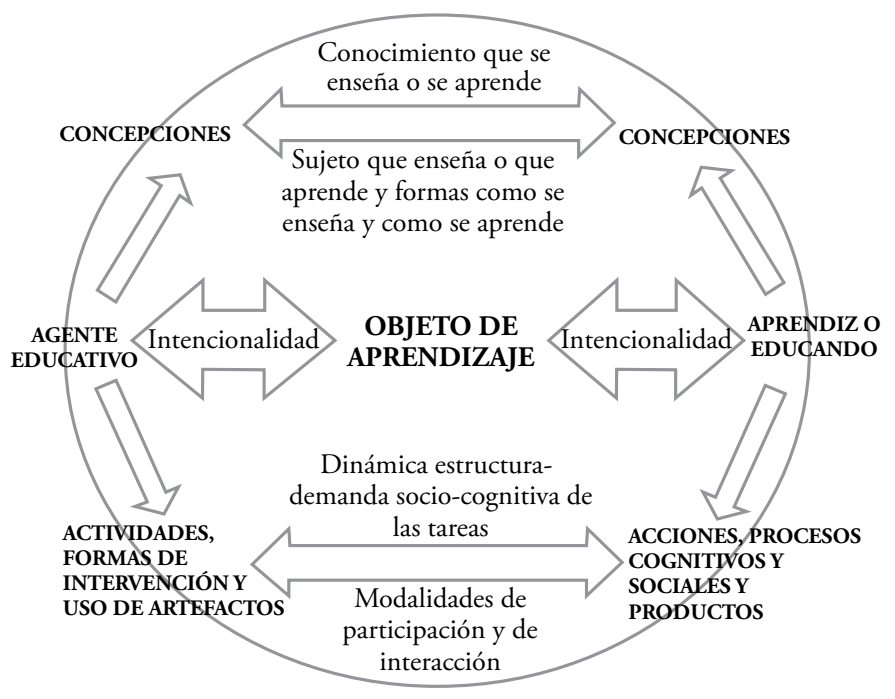

Figura 1. Estructura compleja y dinámica de un ambiente de aprendizaje

En cuanto a sus elementos fundamentales el ambiente de aprendizaje impli$\mathrm{ca}$, en primer lugar, al agente educativo que lo propone y a los aprendices que participan en él. Un ambiente de aprendizaje siempre es generado y organizado por un agente educativo para uno o más educandos. Se denomina agente educativo a quien intencionalmente plantea el conjunto de actividades, acciones y formas de intervención dirigidas a la consecución del objetivo de aprendizaje por parte de los aprendices. El "agente educativo" es la forma genérica para designar a "quien" diseña y dirige la actividad educativa, y los "aprendices" o "educandos" son "quienes" aprenden y se desarrollan. Por lo tanto, el agente educativo no siempre es un profesor y los aprendices no siempre son los nińos de una escuela. La relación agente educativo-educando se puede observar también en una comunidad que dispone actividades culturales para sus miembros, 
una organización o un jefe que genera una serie de acciones dirigidas a mejorar la competencia productiva de sus empleados, una comunidad de pescadores o zapateros que enseñan a los niños a pescar o hacer zapatos, un padre de familia que genera una serie de reglas para el aprendizaje de un hábito en sus hijos, un conjunto de tutores con diferentes niveles de experticia que preparan a nuevos miembros para asumir las responsabilidades de un grupo, etc.

En segundo lugar, todo ambiente de aprendizaje implica como elementos fundamentales: el objetivo central y las metas específicas de aprendizaje, las actividades planteadas por el agente educativo con sus estructuras y demandas cognitivas, y las operaciones, estrategias o desempeños que los aprendices usan para enfrentarse a estas actividades. Cada una de las tareas que se proponen en un ambiente de aprendizaje contiene una meta diferenciada, pero articulada al objetivo central, plantea una estructura diferente y exige el uso de varios funcionamientos mentales en los aprendices que se hacen evidentes en su desempeño. En psicología educativa se conoce a esta relación como estructura-demanda cognitiva de la tarea y determina la complejidad cognitiva de la situación (Otálora, 2009). La estructura de una situación sencilla como, por ejemplo, el dictado de un texto para que los niños copien en el cuaderno exige que se lleve a cabo un proceso de transcodificación literal de un texto en formato verbal a un texto en formato escrito, y este proceso genera como demanda cognitiva el uso de procesos básicos como atención sostenida, memoria a corto plazo y el uso de una regla de traducción de un código a otro. Esta estructura no genera demanda cognitiva compleja como comprensión o uso creativo del conocimiento previo. La estructura de una situación de resolución de problemas, por el contrario, puede tener una demanda cognitiva más compleja en la medida que un individuo tendría que comprender los elementos y las relaciones implicadas en la tarea para buscar estrategias pertinentes y alcanzar la meta. La búsqueda de estrategias exige no sólo el uso de conocimiento sino la comprensión de los contextos en los que éste puede ser usado y de recursos cognitivos más avanzados que la memoria o la atención. Entre mayor nivel de complejidad tenga una tarea más avanzada será la demanda cognitiva y, por ende, las operaciones y estrategias de los aprendices.

En tercer lugar, todo ambiente de aprendizaje comprende los artefactos culturales, utilizados por el agente educativo en las situaciones, con el fin de garantizar el logro de las metas formativas. Entre los artefactos culturales se encuentran: el marco simbólico o contexto, los géneros discursivos y usos del lenguaje, las modalidades de intervención, las ayudas pedagógicas, los materiales, y los lugares y tiempos en los cuales se desarrollan las actividades. El uso de artefactos culturales genera la dinámica de un ambiente de aprendizaje. A través de las 
diferentes modalidades de intervención y los géneros discursivos, los agentes educativos dinamizan las formas de participación de las personas involucradas en la situación y las modalidades de interacción entre el agente educativo-educando, educando-educando o entre educando-objetos de la situación. Estas formas de participación e interacción pueden llegar a ser tan variadas y combinarse de tantas maneras que constituyen uno de los criterios primordiales para determinar la complejidad del espacio educativo.

En cuarto lugar, el ambiente de aprendizaje implica como elementos fundamentales las concepciones e intencionalidades de los agentes educativos y de los educandos. Es importante reconocer que todos los elementos anteriores como las metas de aprendizaje, la estructura y demanda cognitiva de las actividades y los artefactos culturales son propuestos por el agente educativo de acuerdo con sus concepciones sobre el desarrollo y el aprendizaje de los educandos, sobre el conocimiento que se pretende enseñar, sobre la forma como se debe enseñar y sobre los fines de la educación. Las concepciones dirigen las acciones del agente educativo, tanto como sus intenciones, y de esta manera constituyen un elemento fundamental para la dinámica del ambiente de aprendizaje. Pero, también, los educandos aportan a esta dinámica a través de sus concepciones sobre las intenciones y las formas de enseñar del agente educativo, sobre el conocimiento que aprenden y las metas de la educación. Por supuesto, los aprendices también construyen concepciones sobre su propio aprendizaje.

Las relaciones que se establecen entre los elementos fundamentales de un ambiente de aprendizaje permiten concebirlo no sólo como un espacio de construcción de conocimiento estructurado, sino como un escenario complejo y dinámico que cambia en el tiempo, que es flexible a las necesidades de los educandos o de los agentes educativos, que se puede enriquecer. La dinámica de un ambiente de aprendizaje puede variar en función de metas emergentes que afectan el conjunto de actividades de los agentes educativos o las acciones de los educandos (Coll y Onrubia, 1996). Sin embargo, la dinámica de un ambiente de aprendizaje puede ser predecible y controlada sin dejar de ser flexible y pertinente para la consecución del objetivo de aprendizaje central para el aprendiz o el grupo de educandos.

\section{Desarrollo infantil y espacios educativos significativos}

Un espacio educativo se puede considerar significativo en la medida que promueva el principal sentido de la educación: el aprendizaje y el desarrollo humano. Desde este punto de vista es pertinente revisar algunas consideraciones sobre la concepción del desarrollo infantil que permitan derivar implicaciones 
para el diseño de ambientes de aprendizaje que contribuyan, significativamente, al crecimiento integral de los nińos. A partir de estas implicaciones es posible definir la naturaleza de los espacios educativos significativos.

La primera consideración es que los nińos poseen competencias innatas y las usan para desarrollar nuevas competencias. De acuerdo con algunos autores, los bebés vienen equipados con mecanismos innatos en diferentes dominios de conocimiento, como el social, lingüístico, físico ó matemático, que son utilizados para atender y organizar la información que proviene del medio y comprender su realidad (Karmiloff-Smith, 1996; Gelman, 2000). El desarrollo infantil consiste en un proceso de reorganizaciones y transformaciones permanentes de estas y de nuevas competencias, y por lo tanto, requiere escenarios exigentes que revelen los retos del mundo real. Desde el nacimiento y durante el período de la infancia son diversos los espacios donde los niños actúan e interactúan con otros y con su mundo. Los diálogos madre-bebé durante el baño diario, la resolución de un problema cotidiano como amarrarse los zapatos, encontrar un juguete perdido o ayudar a los hermanos a lavarse las manos, las prácticas tradicionales como armar el pesebre o construir un caballito de palo y los juegos de competencia, como "La gallina ciega" o "La rayuela" con los compañeritos constituyen situaciones enriquecedoras y novedosas para los niños porque los enfrentan a las exigencias de la vida diaria.

Sin embargo, no toda experiencia durante la infancia resulta significativa para los niños. El desarrollo infantil no se traduce sólo en aprender "contenidos" sobre el mundo o generar "hábitos" para sobrevivir. Por esta razón las actividades centradas en el uso de rutinas ya aprendidas, como por ejemplo, hacer planas y bolitas de papel para pegar en un cuaderno, copiar dictados o jugar todos los días el mismo juego, no constituyen para los niños un desafío porque no hay nada nuevo que crear. Igualmente las actividades centradas en el uso exclusivo de la memoria y la atención como aprender un texto para hacer una exposición o poner atención al tablero mientras la maestra habla no favorecen el desarrollo del pensamiento creativo ni la autonomía en la resolución de problemas para la vida. Un espacio educativo significativo debe ser, por el contrario, un escenario de aprendizaje retador y generador de múltiples experiencias para quienes participan en él. Un espacio educativo resulta significativo para el desarrollo en la infancia cuando el conjunto de situaciones relacionadas entre sí, en el ambiente de aprendizaje, favorecen la construcción de nuevo conocimiento y permiten el crecimiento de formas de pensamiento más avanzadas y modalidades más complejas de interacción (Otálora, 2007). Esta constituye, en esencia, la función de la educación en la sociedad. 
Una segunda consideración sobre la concepción de desarrollo infantil es el reconocimiento del rol activo que los nińos desepeñan en sus propios procesos de cambio. Ellos piensan porque les gusta pensar y conocen porque quieren saber siempre más, exploran porque quieren descubrir, deciden cuándo quieren aprender algo nuevo y además deciden qué aprender. Más sorprendente aún, los niños vuelven una y otra vez sobre lo que ya saben para comprender mejor (Karmiloff-Smith, 1996). Ellos igualmente son capaces de generar nuevas formas de interactuar con los adultos, con los otros nińos, con las cosas y con los eventos. Son capaces de organizar información compleja y de articular sus conocimientos y recursos cognitivos en procedimientos dirigidos a metas, generando así estrategias para solucionar problemas (Otálora, 2007). Además, pueden generar diversas maneras de enfrentarse a las tareas y situaciones cotidianas, y responder a sus demandas, en un mismo momento de su desarrollo (Siegler, 2007). Por esta razón, un espacio educativo significativo es aquel que promueve la actuación de los nińos en el mundo y la autonomía sobre sus procesos de aprendizaje. Un espacio educativo en el que el adulto es el que piensa y el nińo solo sigue órdenes, en el que el niño no puede tomar algunas decisiones, en el que sólo puede utilizar una única forma de hacer las cosas considerada la correcta, en el que no puede probar estrategias y el error significa que no sabe, o en el que todos los días hace las mismas cosas, no es considerado significativo porque no le permite pensar, saber, descubrir siempre más. Por el contrario, un espacio educativo es significativo cuando los niños pueden resolver problemas por sí mismos, con apoyo de sus hermanos o padres, compañeros o maestros, en el que pueden tomar sus propias decisiones, aprender del fracaso y el error y utilizar sus resultados efectivos para resolver nuevos problemas en contextos diferentes.

Una tercera consideración es que los niños no aprenden ni se desarrollan de la misma manera. El desarrollo infantil no es lineal, no tiene un inicio y un final fijos, ni sigue una vía de avance progresivo de menos a más conocimiento, y por lo tanto, sus cambios no dependen exclusivamente de la edad (Puche-Navarro, 2003). Por esta razón cuando los ambientes de aprendizaje son significativos no están relacionados con una edad determinada en la que los nińos deban participar, sino que permiten la inclusión de bebés, infantes, niños mayores e incluso adultos en la consecución de las metas de las actividades, como ocurre en la vida real. Es posible, por ejemplo, que en un juego que enseña una maestra a sus niños, sean ellos quienes a veces expliquen las instrucciones a sus compañeros o elijan los roles del juego, y esta no sea una labor exclusiva del adulto. Igualmente, es posible que la maestra juegue, y no sean sólo los nińos quienes pueden jugar. Los espacios educativos significativos no presentan tampoco una sola manera de 
hacer las cosas, por el contrario favorece múltiples vías de resolución. Situaciones cerradas como hacer una plana o decir la capital de un país presenta una única respuesta correcta. Enfrentarse a problemas cotidianos como ayudarle a hacer el mercado a la mamá, organizar los juguetes del hogar comunitario o explorar las ideas del abuelo sobre la navidad son abiertas e implican varias formas de alcanzar las metas.

Una cuarta consideración es que los niños no aprenden ni se desarrollan solos. La pertenencia a un grupo cultural en el que se construyan relaciones entre los miembros que participan en él, juega también un papel importante en el desarrollo infantil, porque afecta de manera única y diferenciada la experiencia de los niños, enriqueciéndola y colmándola de importantes significados. Los niños requieren de la presencia de otros nińos que los acompañen en la empresa de crecer, y de adultos que apoyen sus procesos de cambio. Esta interacción favorece la construcción de su identidad y la formación de rasgos específicos de su personalidad que los diferencian de los demás. Además, los niños establecen relaciones también con los objetos del contexto, desde explorar cómo funcionan las cosas y el papel que cumplen en una situación dada, hasta la constitución de esos objetos como herramientas para su comunicación o su supervivencia. Finalmente, la introducción de los nińos en la consecución de metas legítimas de su grupo cultural dirige el aprendizaje hacia modos cada vez más adecuados de participación en las actividades de supervivencia y de convivencia, y a la generación de una amplia red de saberes compartidos. Así, de manera autónoma, los niños logran alimentar el sentido de pertenencia a su comunidad y ocupar un lugar central y específico en ella. A partir de estas consideraciones, emerge como necesidad primaria el diseño, o mejoramiento, de espacios educativos que reconozcan la relevancia de los contextos y las tramas culturales en las cuales los niños caracterizan su individualidad, tejen sus primeros vínculos sociales y despliegan sus competencias.

Como última consideración, es necesario señalar que estas formas de pensamiento y aproximaciones de los niños a la realidad, sólo se pueden evidenciar en las conductas y verbalizaciones que ellos muestran cuando manipulan los objetos, cuando juegan, cuando hacen una pregunta, cuando realizan sus actividades cotidianas o cuando interactúan con los demás. Los psicólogos llaman desempeños a este conjunto de verbalizaciones y procedimientos. Por lo tanto, los espacios educativos son significativos cuando permiten a los agentes educativos observar, describir y analizar fácilmente los desempeños de los niños en las actividades que les proponen y hacer seguimiento a la manera como éstos cambian a través del tiempo. En otras palabras, un espacio educativo significativo es aquel que 
facilita la observación y el análisis de indicadores de desarrollo que dan cuenta de las competencias de los niños en los diferentes dominios de conocimiento.

Estas consideraciones permiten concluir que un espacio educativo es significativo si se trata de cualquier situación, actividad, tarea o práctica que sea acompañada por pares y cuidadores y enriquecida con la generación de múltiples interacciones, que se relacione con otras experiencias de la vida de los niños y con las metas del grupo cultural al que pertenecen, que les brinde la oportunidad de aprender conocimiento realmente nuevo, establecer desafíos, resolver problemas complejos, descubrir, crear, innovar y que les exija "pensar". Desde este punto de vista, los espacios educativos significativos son ambientes de aprendizaje que favorecen no sólo la adquisición de múltiples 'saberes', sino que fortalecen las competencias afectivas, sociales y cognitivas necesarias para enfrentar de manera creativa las demandas crecientes del entorno durante los primeros años de vida.

\section{Criterios para diseñar o enriquecer espacios educativos significativos}

Teniendo en cuenta la definición de ambientes de aprendizaje, la concepción de desarrollo en la primera infancia y las implicaciones para la implementación de espacios educativos retadores y generadores de múltiples experiencias, es necesario explicitar y operacionalizar cinco criterios que hacen que un ambiente de aprendizaje se constituya en un espacio educativo significativo. Esos criterios piden que ese espacio sea una situación: 1) estructurada, alrededor de objetivos centrales y metas específicas, 2) intensiva, que exija la resolución de problemas relacionados con metas de la cultura, 3) extensiva, que permita manipular la complejidad de las metas en el tiempo, 4) que favorezca contextos complejos de interacción y, 5) generativa, que exija el uso de variadas competencias. La figura 2 esquematiza los criterios que pueden y deben estar presentes en una misma situación de manera articulada.

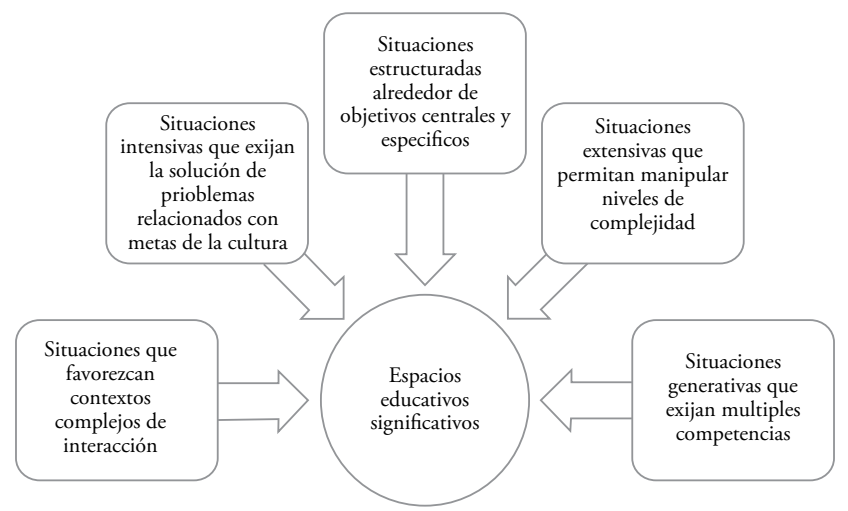

Figura 2. Criterios que definen un espacio educativo significativo 
El uso de prácticas cotidianas y culturales es considerada una estrategia pertinente para lograr la comprensión y el fortalecimiento de las competencias sociales y cognitivas de los niños, relevante para sus vidas, aún como miembros de grupos culturales específicos (Orozco, et al, 2001; Otálora, 2007); y, por lo tanto, permiten la implementación de cada uno de estos criterios. Por esta razón, la revisión de los criterios se aborda ilustrando el uso de prácticas culturales diseñadas por agentes educativos, que participan en dos programas de intervención desarrollados desde la psicología educativa por investigadores del Centro de Investigaciones en Psicología de la Universidad del Valle -uno en el departamento del Valle, y otro en el departamento del Cauca-Colombia.

\section{Criterio 1: situaciones estructuradas alrededor de objetivos centrales y especificos}

El primer criterio para definir un espacio educativo como significativo es que se traduzca en una situación estructurada. Una situación es estructurada cuando cada una de sus actividades se dirige a metas de aprendizaje específicas y éstas, a su vez, giran alrededor de un objetivo de aprendizaje amplio. Los objetivos son el punto de partida para diseñar u organizar las actividades y ambos constituyen componentes fundamentales que brindan estructura a una situación porque permiten configurar la secuencia completa o unidad de aprendizaje. Un tercer componente en esta estructura es a veces olvidado, pero es igual de importante. La temática, también llamada tópico, contexto o marco simbólico, es necesaria porque facilita la traducción de objetivos y metas al conjunto de actividades de la situación y posibilita la articulación de estas actividades entre sí.

Dos actividades encontradas en un hogar comunitario del Distrito de Aguablanca del departamento del Valle-Colombia ilustran situaciones no estructuradas. Un agente educativo pide a sus nińos de 2 a 6 ańos jugar a la ronda del "Pan y canela" todos los días, y los deja jugando solos hasta que se aburran o se cansen. Cuándo se le indaga por el objetivo de esta actividad el agente educativo plantea que los niños se divierten y así los puede tener ocupados y entretenidos, mientras él hace otra tarea. Además ya se la saben porque la juegan todos los días. Esta actividad muestra una situación con sentido sólo para el agente educativo ("puede hacer otras tareas") pero no existe un propósito de aprendizaje para los participantes del juego por tres razones: 1) el único propósito es estar "entretenido" 2) no hay construcción de nuevo conocimiento ni se promueve la creatividad y el uso del pensamiento, dado que diariamente los niños emprenden la misma actividad que conocen de memoria, y 3) los niños no tienen el acompańamiento de un cuidador que observe sus acciones y verbalizaciones interpretándolas en 
términos de su desarrollo y que, a su vez, manipule la situación para lograr que ellos avancen en este desarrollo.

El mismo agente educativo pide en otro momento a los niños más grandecitos hacer una plana del 2, una del 3 y una del 4 con el propósito de aprender el significado de los números. Aunque esta actividad si tiene un propósito de aprendizaje, éste no es coherente con el tipo de conocimiento que se pretende enseñar -los números-, ni con la forma como los niños aprenden este conocimiento -conteos y composiciones aditivas y multiplicativas. Se trata de una actividad rutinaria que permite fortalecer la habilidad grafo-motora, pero no tiene relación con la creación de significados del dos, el tres y el cuatro y mucho menos con la idea de alcanzar un nivel de comprensión abstracta de ellos.

Este agente educativo tiene sin embargo dos fortalezas. Usa una práctica tradicional en la primera actividad y tiene un objetivo de aprendizaje específico en la segunda actividad. En el programa de intervención el agente educativo logra crear un ambiente de aprendizaje estructurado para trabajar competencias matemáticas y sociales relevantes para el desarrollo de sus nińos. Plantea como objetivo amplio lograr que ellos construyan significados abstractos de los números del 1 al 12 en una situación de trabajo en equipo. Para esto revisa varias prácticas culturales de la comunidad que no usa rutinariamente con los nińos y que, potencialmente, puedan facilitar la consecución de este objetivo amplio. Elige la ronda "Agua de limón", y organiza dos ciclos de cuatro actividades, cada una con una meta de aprendizaje diferente en función de las competencias sociales o matemáticas que quiere promover. La práctica se implementa con los doce niños del hogar comunitario.

\begin{tabular}{|c|c|}
\hline Secuencia de actividades por ciclo & Metas específicas por competencia \\
\hline $\begin{array}{l}\text { Actividad 1. Aprender el estribillo de la ronda } \\
\text { y los pasos } \\
\text { Los niños hacen un círculo y empiezan a girar hacia } \\
\text { un lado cantando: "Agua de limón, vamos a jugar, } \\
\text { el que quede solo, solo quedará. ¡Hey!". Los niños } \\
\text { cambian de dirección su cuerpo y giran hacia el } \\
\text { lado contrario cantando de nuevo el estribillo. Al } \\
\text { finalizar la persona que dirige el juego expresa en } \\
\text { voz alta "En grupos de..." y dice un número. Los } \\
\text { niños deben organizar grupos con el número de } \\
\text { jugadores que ha sido indicado. }\end{array}$ & $\begin{array}{l}\text { Competencia social: } \\
\text { Para trabajar en equipo los niños requieren compren- } \\
\text { der y manejar las reglas de la actividad en la que par- } \\
\text { ticipan de manera conjunta. Al aprender el estribillo } \\
\text { de ésta ronda los niños infieren las reglas del juego y } \\
\text { comprenden que cuando cantan "El que quede solo, } \\
\text { solo quedará" están explicitando la regla fundamental } \\
\text { del juego "El que quede solo, pierde, para ganar hay } \\
\text { que hacer grupos", y cuando dicen "hacer grupos de } \\
\text { tres" están explicitando la regla especifica de organi- } \\
\text { zación de los grupos "si no hace ese número exacto, } \\
\text { pierde, hay que hacer grupos de tres". }\end{array}$ \\
\hline
\end{tabular}




\begin{tabular}{|l|}
\hline \multicolumn{1}{|c|}{ Secuencia de actividades por ciclo } \\
\hline Actividad 2. Jugar a la ronda conformando \\
grupos de tres jugadores \\
El número que el agente educativo da es el tres y \\
los niños deben conformar los grupos. El agente \\
educativo pide a los niños que cuenten para \\
llegar al tres. Una vez conformados los grupos \\
pide de nuevo que se cuenten y si hay más sa- \\
quen los jugadores que sobran y si hay menos \\
busquen otro u otros jugadores hasta completar \\
el número indicado.
\end{tabular}

\section{Actividad 3. Sumar grupos de tres}

Cuando los nińos terminan de hacer grupos de tres el agente educativo les pregunta: "aquí hay un grupo de tres niños, y aquí hay otro grupo de tres niños, ¿cuántos niños hay en total?". Después de que los nińos contestan, el agente educativo interviene para que los niños lleguen al resultado correcto y después pregunta: "y si tengo otros tres niños, ¿̇uántos hay?". Se hacen las mismas preguntas e intervenciones hasta terminar todos los grupos de tres.

\section{Actividad 4. Multiplicar grupos de tres}

Cuando los niños terminan de sumar los grupos de tres el agente educativo les hace la siguiente pregunta: "bien, si hay dos grupos y cada uno tiene tres niños, ¿̇cuántos niños hay en total.?" Después de que los niños contestan, el agente educativo interviene para que los nińos lleguen al resultado correcto y después formula la siguiente pregunta: "y si tengo tres grupos y cada uno tiene tres niños, ¿̨cuántos niños hay en total?". Se hacen las mismas preguntas e intervenciones hasta terminar todos los grupos de tres.

\section{Metas específicas por competencia}

\section{Competencia matemática:}

Comprender el número tres como una totalidad (cardinal) y comprender que el conteo puede ser usado para conformar esta totalidad. Si el niño no tiene un significado para tres deberá construirlo a través del uso del conteo y haciendo explícito el número total de niños del grupo.

\section{Competencia social:}

Manejo de las reglas del juego y trabajo en equipo. Los grupos no se pueden organizar sin la participación de todos, los niños más grandes apoyan a los más pequeños para organizar los grupos de acuerdo al número que se ha expresado en voz alta, contándoles y mostrándoles cuánto es "tres" y de ninguna manera quedarse fuera de un grupo.

Este no es un paso de la ronda tradicional pero constituye una actividad de enriquecimiento del ambiente de aprendizaje.

\section{Competencia matemática:}

Desarrollar composiciones aditivas que permitan establecer nuevas totalidades como "seis", "nueve", "doce", construyendo significados abstractos de estos números.

\section{Competencia social:}

Trabajo en equipo para llegar a los resultados de la tarea a través de conteos o sumas conjuntas.

Este tampoco es un paso de la ronda tradicional pero constituye otra actividad de enriquecimiento del ambiente de aprendizaje.

\section{Competencia matemática:}

Desarrollar composiciones multiplicativas que permitan establecer nuevas totalidades como "seis", "nueve", "doce", construyendo significados abstractos de estos números.

Los niños comprenderán al final que los números implican relaciones unitarias (a través del conteo) y relaciones aditivas y multiplicativas (a través de las sumas y las multiplicaciones accediendo a su naturaleza abstracta).

\section{Competencia social:}

Trabajo en equipo para llegar a los resultados de la tarea a través de conteos, correspondencias uno-tres, sumas o multiplicaciones conjuntas.

Este ciclo se repite de nuevo pero complejizando la demanda de las actividades y para ello trabaja con el número cinco. De esta manera, logra trabajar la construcción de significados abstractos de varios números como el 3, 6, 9, 12, 5,10 y 2. Este ambiente de aprendizaje ilustra la manera como un conjunto de actividades se articulan entre sí en función de un propósito amplio de aprendizaje 
y metas específicas de comprensión, vinculadas al desarrollo de competencias sociales y matemáticas, utilizando como contexto una práctica cultural tradicional en Colombia y formas de enriquecimiento de esta práctica.

\section{Criterio 2: situaciones intensivas de resolución de problemas relacionados con la cultura}

Son llamadas intensivas las situaciones que plantean a las personas la resolución de uno o varios problemas y que, por lo tanto, exigen de ellas una actividad mental más intensa que otro tipo de tareas, como por ejemplo, las rutinas. De acuerdo con Luria y Tsvetkova (1981) un problema es una pregunta a la que es imposible dar una respuesta inmediata y que determina la actividad posterior de las personas que se enfrentan a ella. Esta actividad posterior llamada "resolución" tiene un carácter selectivo dado que se apoya en un programa lógico de operaciones relacionadas entre sí. Cuando las personas se enfrentan al problema, deben analizar la información contenida en la pregunta, seleccionar los datos esenciales, conocidos o desconocidos y confrontarlos. A partir de esta orientación las personas generan un esquema de resolución llamado estrategia y desencadenan las operaciones que conducirán exitosamente a la respuesta buscada. Para los autores, ésta representa la estructura característica de la actividad intelectual de las personas. Desde este punto de vista, la resolución de problemas constituye un escenario privilegiado para el desarrollo de los nińos ya que la búsqueda de la respuesta les permitirá usar de manera intensa su pensamiento.

Newell y Simon (1972) muestran la relación entre la estructura general de un problema y la demanda cognitiva de la resolución. Según los autores, una situación de resolución de problemas tiene un estado inicial; un estado final deseado, también llamado meta; una barrera entre los dos estados y una serie de pasos u operaciones necesarias para pasar de uno al otro venciendo la barrera. Los autores proponen dos subprocesos básicos para la resolución de un problema denominados comprensión y búsqueda. Por una parte, la comprensión del problema exige a las personas la identificación de una red de relaciones en el planteamiento del estado inicial haciendo uso de su conocimiento previo, y el razonamiento sobre estas relaciones, el cual lleva a la ampliación de la red creando nuevo conocimiento. Por otra parte, el proceso de búsqueda lleva a la generación de estrategias que se manifiestan en procedimientos dirigidos a alcanzar el estado meta. Desde esta perspectiva, la resolución de problemas implica a los niños hacer uso de su conocimiento previo para establecer relaciones sobre su realidad, enriquecer esta red de relaciones y articular sus recursos mentales y emocionales en estrategias que permitan alcanzar la respuesta buscada de manera 
efectiva -respuesta correcta- y eficiente -con el menor gasto de recursos-. Por estas razones, la resolución de problemas constituye un espacio de aprendizaje ideal en contextos educativos que privilegien la comprensión del mundo más que la memorización de contenidos sobre él, y la transformación estratégica del mundo más que la recepción pasiva de conocimiento.

Las prácticas cotidianas son situaciones que fácilmente pueden ser utilizadas por los agentes educativos como situaciones de resolución de problemas, pues son actividades que tienen un carácter práctico que implica a los niños aprender a responder a las necesidades del entorno. Las prácticas cotidianas son actividades diarias compartidas por una gran parte de los miembros de la comunidad, que siguen siendo vigentes para la consecución de metas relevantes de supervivencia como por ejemplo la socialización, la convivencia, la alimentación, la salud, la higiene y la consecución de recursos económicos. La participación en las actividades cotidianas está en función de propósitos prácticos dirigidos a cubrir estas necesidades básicas. Involucrarse por primera vez en actividades como saludar a los compañeros cada mañana, ayudar a servir el almuerzo, organizar la casa o el salón de clases y preparar colaborativamente la merienda puede llegar a representar un problema para los niños, frente al cual ellos pueden espontáneamente articular sus conocimientos, hipótesis y recursos de pensamiento para implementar los procedimientos que la situación requiere. A su vez, el análisis de las conductas o actividad de los niños permite a los agentes educativos analizar sus competencias y utilizar esta información para ayudarlos a desarrollar nuevas competencias.

Sin embargo, por su naturaleza una situación de resolución de problemas debe ser siempre una situación novedosa. Si los niños se enfrentan a la misma actividad todos los días se convierte en una rutina y deja de ser un problema para ellos. Por ejemplo, cuando los nińos empiezan a vestirse solos después del primer año de vida, enfrentan un verdadero problema porque la meta -estar vestido- está mediada por obstáculos que ellos deben superar como la coordinación de los movimientos de ambas manos con cada parte de la ropa y con cada parte del cuerpo. Una vez los nińos dominan los movimientos que deben realizar con la ropa y con su cuerpo, cuando se enfrentan nuevamente a la tarea de hacerlo, ya no representa un problema, ya no hay aprendizaje, ya no les exige pensar, incluso, ya no es necesario el uso de la conciencia para llegar a la meta efectivamente. Por estas razones, los espacios educativos significativos requieren la implementación de actividades novedosas, que impliquen retos, no sólo motores y espaciales, sino del pensamiento. Así, el uso de prácticas cotidianas no quiere decir el uso de rutinas aprendidas, sino el aprovechamiento de actividades novedosas en las que cotidianamente los niños se pueden involucrar para solucionar estratégicamente problemas. 
Por otra parte resulta necesario vincular, en los ambientes de aprendizaje, metas relevantes de la cultura. Gran parte de las actividades utilizadas en los espacios educativos son descontextualizadas de los intereses de las comunidades a las que los niños pertenecen y, en esta medida, se olvidan rápidamente porque no son útiles para la vida o interesantes para ellos. Brown, Collins y Duguid (1989) plantean que la cognición es específica de la situación en que se aprende y deriva del vínculo de la acción de los sujetos con el contexto. Además, señalan que el conocimiento cultural está distribuido en el seno de las comunidades, no pertenece a sujetos individuales. Es por esta razón que los contenidos, temas o tópicos de los espacios educativos se deben buscar en las metas de estas comunidades, deben promover el acceso al saber propio de sus miembros, negociar significados y garantizar la acción de todos los participantes sobre el contexto. De esta manera los nińos construirán no sólo conocimiento en dominios específicos, sino que fortalecerán si identidad cultural y su sentido de pertenencia. El Diseño de la huerta comunitaria es una práctica construida por tres maestras de preescolar de una escuela rural del departamento del Cauca-Colombia, durante un programa de intervención realizado desde la psicología educativa. Esta práctica permite ilustrar la vinculación de metas de su comunidad en situaciones de resolución de problemas y, a la vez, promover competencias sociales, matemáticas, comunicativas y científicas. Esta secuencia de actividades corresponde a la primera fase de un proyecto comunitario más amplio que implica también la siembra, cuidado y cosecha en la huerta.

\begin{tabular}{|c|c|}
\hline Secuencia de actividades & Metas específicas por competencias \\
\hline $\begin{array}{l}\text { Actividad 1. Planificación de la huerta } \\
\text { comunitaria } \\
\text { Las maestras introducen el proyecto comunitario } \\
\text { con las siguientes preguntas: ¿han conocido algu- } \\
\text { na vez una huerta?, ¿qué se hace en ella?, ¿qué se } \\
\text { puede sembrar en una huerta? Después indagan } \\
\text { sobre el significado de huerta comunitaria pre- } \\
\text { guntando: ¿para qué creen que sirve una huerta } \\
\text { comunitaria? o ¿quiénes van a obtener beneficio } \\
\text { de una huerta comunitaria? Las maestras utilizan } \\
\text { las ideas previas de los niños, las complementan } \\
\text { y generan una definición de huerta comunita- } \\
\text { ria que escriben en un cartel grande. Después } \\
\text { preguntan: ¿cuáles son los pasos que tendrían } \\
\text { que seguir para construir una huerta en la que } \\
\text { se pueda sembrar frijol, maíz y cilantro? Las } \\
\text { respuestas de los niños son completadas con } \\
\text { ideas de las maestras y escriben estos pasos en el } \\
\text { cartel. Al finalizar, este es colgado en una pared } \\
\text { para que todos puedan ver la definición y los } \\
\text { pasos a seguir. }\end{array}$ & $\begin{array}{l}\text { Competencia social: } \\
\text {-Identificación de una meta pertinente para su } \\
\text { comunidad de origen. } \\
\text {-Participación en la planificación de un proyecto } \\
\text { comunitario. } \\
\text {-Toma de decisiones. } \\
\text { Competencia comunicativa: } \\
\text {-Argumentación. } \\
\text {-Negociación de significados. } \\
\text {-Comprensión de la idea central de un texto. } \\
\text {-Producción conjunta de un texto. } \\
\text { Competencia científica: } \\
\text {-Formulación de hipótesis. } \\
\text {-Planificación de un evento. }\end{array}$ \\
\hline
\end{tabular}




\begin{tabular}{|c|c|}
\hline Secuencia de actividades & cíficas por competencias \\
\hline $\begin{array}{l}\text { Actividad 2. Diseño de un plano de la } \\
\text { buerta comunitaria } \\
\text { Los niños son llevados a un terreno amplio en } \\
\text { el que quedará ubicada la huerta. Las maestras } \\
\text { organizan grupos de cuatro nińos cuidando } \\
\text { que hayan de diferentes edades y a cada grupo } \\
\text { le asignan una porción del terreno con medidas } \\
\text { específicas señalado por estacas en las esquinas. } \\
\text { Las maestras entregan a cada grupo un diario } \\
\text { de registro previamente diseñado, y piden a los } \\
\text { niños que realicen allí un plano de la porción } \\
\text { de la huerta que les dieron, en el cual deben } \\
\text { distribuir el espacio en tres triángulos, rectán- } \\
\text { gulos o cuadrados de tal manera que puedan } \\
\text { ser sembrados los tres productos. Las maestras } \\
\text { llaman eras a estos espacios. }\end{array}$ & $\begin{array}{l}\text { Competencia social } \\
\text {-Trabajo en equipo para lograr una meta del } \\
\text { grupo. } \\
\text {-Participación en la ejecución de un proyecto } \\
\text { comunitario. } \\
\text {-Toma de decisiones. } \\
\text { Competencia comunicativa } \\
\text {-Socialización de ideas y resultados. } \\
\text {-Negociación de significados. } \\
\text { Competencia cientifica } \\
\text {-Formulación de hipótesis. } \\
\text {-Planificación de un evento. } \\
\text { Competencia matemática } \\
\text {-Uso de relaciones espaciales para la represen- } \\
\text { tación bidimensional de un espacio tridimen- } \\
\text { sional. } \\
\text {-Uso de relaciones geométricas para la distribu- } \\
\text { ción de la porción del terreno en el plano. } \\
\text {-Identificación de figuras geométricas. }\end{array}$ \\
\hline $\begin{array}{l}\text { Actividad 3. Distribución de la huerta } \\
\text { Las maestras entregan a los grupos cuatro palos } \\
\text { (o uno por niño) de igual medida que llaman } \\
\text { estacas. Piden a los niños medir tanto el terreno } \\
\text { completo como la parte cercada haciendo uso } \\
\text { de la estaca o haciendo uso de partes del cuerpo, } \\
\text { como el pie. Después se les pide distribuir en su } \\
\text { porción de terreno los tres espacios o eras para } \\
\text { sembrar cada producto. Las maestras preguntan: } \\
\text { ¿qué se puede utilizar para medir el terreno?, } \\
\text { ¿cómo pueden medir con la estaca?, ¿̇o con el } \\
\text { pie? De acuerdo con las respuestas, los niños } \\
\text { empiezan a medir el terreno con lo que eligió } \\
\text { cada grupo y a distribuir el espacio. Algunas de } \\
\text { las preguntas formuladas por las maestras para la } \\
\text { sistematización de los datos que los niños hacen } \\
\text { de la actividad son: ¿cuántas cuartas (estacas, } \\
\text { pies, etc.) mide el terreno?, ¿cuántas cuartas } \\
\text { (estacas, pies, etc.) mide la era?, ¿en una estaca } \\
\text { cuántas cuartas hay?, ¿en una estaca cuántos } \\
\text { pies hay?, ¿cuántas cercas del mismo tamaño } \\
\text { puedes hacer en el terreno?, ¿qué formas tienen } \\
\text { las eras? Todas las respuestas de los niños deben } \\
\text { ser anotadas en su diario de registro. } \\
\text { Cuando los niños terminan de distribuir el te- } \\
\text { rreno deben cercarlos con cuerdas largas que los } \\
\text { niños deben amarrar a las estacas de las esquinas. }\end{array}$ & $\begin{array}{l}\text { Competencia social } \\
\text {-Trabajo en equipo para lograr una meta del } \\
\text { grupo. } \\
\text {-Participación en la ejecución de un proyecto } \\
\text { comunitario. } \\
\text {-Toma de decisiones. } \\
\text { Competencia comunicativa } \\
\text {-Socialización de ideas y resultados. } \\
\text {-Negociación de significados. } \\
\text {-Sistematización escrita de la información en el } \\
\text { diario de registro. } \\
\text { Competencia científica } \\
\text {-Formulación de hipótesis. } \\
\text { Competencia matemática } \\
\text {-Comprensión de los fines de la medición. } \\
\text {-Medición con patrones de medidas no con- } \\
\text { vencionales. } \\
\text {-Uso de relaciones espaciales para la transforma- } \\
\text { ción de un espacio tridimensional siguiendo un } \\
\text { modelo bidimensional. } \\
\text {-Uso de relaciones geométricas para la distribu- } \\
\text { ción de la porción del terreno en tres eras. } \\
\text {-Comprensión de figuras geométricas. } \\
\text {-Cuantificación. }\end{array}$ \\
\hline
\end{tabular}

Una característica importante de esta actividad es que propone diferentes situaciones de resolución de problemas, como la planeación conjunta de la actividad, la elaboración del plano, la distribución de las eras, la medición de las eras, y la transformación de las unidades de medida, alrededor de la solución 
de un problema más amplio pertinente para el grupo cultural: el diseño de una huerta comunitaria. Además, los agentes educativos permiten a los nińos utilizar sus propios procedimientos -acciones y verbalizaciones- y poner en juego sus propias ideas y conceptos, así estos no sean todavía formales. La actividad está completamente basada en situaciones abiertas que posibilitan diversas formas de resolver los problemas que se les van proponiendo continuamente. Las vías de resolución que los niños utilizan son válidas porque son propias y evidencian sus competencias. A través de la actividad las maestras logran identificar la comprensión alcanzada por los niños y complementan o retroalimentan sus ideas iniciales para generar comprensiones más avanzadas. Esta actividad además les permite plantear nuevos objetivos y retos que son formulados en un segundo ambiente de aprendizaje que hace parte del mismo proyecto comunitario: la siembra de los productos. Así, este tipo de actividades promueve la creación de nuevo conocimiento en los nińos y a la vez, esta creatividad les permite la transformación de su entorno. Es de esta manera, que las situaciones de resolución de problemas constituyen escenarios que exigen a los niños comprometerse de una manera activa con el mundo.

\section{Criterio 3: situaciones extensivas que permitan manipular niveles de complejidad}

Las actividades extensivas son situaciones que además de ser intensivas pueden repetirse en episodios consecutivos -intrasesión- y/o presentarse en múltiples ocasiones a lo largo del tiempo -multisesiones. El objetivo de plantear una situación como extensiva es que los agentes educativos logren observar y diagnosticar el conocimiento que los niños han construido en situaciones intensivas reiteradas, intervenir adecuadamente y llevar un seguimiento de las estrategias empleadas y los avances relativos al objetivo y las metas de aprendizaje.

Un principio de la construcción de conocimiento que sustenta la actividad extensiva como práctica educativa es que el conocimiento de los nińos no es estático, sino que evoluciona permanentemente (Steffe, 1990). Por esta razón, para que una situación intensiva pueda hacerse extensiva, debe contemplar diversos niveles de complejidad, en función de la comprensión observada en los niños. Este elemento es quizá uno de los más importantes en la implementación de estas situaciones, pues manipular niveles de complejidad implica determinar de manera continua, la comprensión que los niños logran a medida que se enfrentan a las actividades propuestas, así como modificar las metas de comprensión planteadas. Este objetivo se logra porque ellos pueden establecer relaciones cada vez más ricas y formales que les permitan avanzar sobre su propio aprendizaje. Ejemplo de una situación intensiva que se vuelve extensiva es la ronda "Agua 
de limón" (ver criterio 1), en la cual el agente educativo manipula el nivel de complejidad de dos maneras, la actividad 2 de la situación exige el uso de la cardinalidad (establecer totales abstractos) y la cuantificación y, a partir de allí, genera otras situaciones cada vez más complejas en función de la relación aritmética a establecer por los niños, una para usar relaciones aditivas y otra para usar relaciones multiplicativas. Además, el agente educativo manipula otro criterio de la complejidad de la situación, el rango numérico, utilizando el primer ciclo para trabajar composiciones aditivas y multiplicativas con el número tres, y el segundo ciclo con el número cinco.

La naturaleza extensiva de esta situación permite la movilización y confrontación del conocimiento de los niños de manera continua. Por esta razón, implementar este tipo de actividad permite a los agentes educativos intervenir adecuadamente a partir de línea de base de los aprendices y hacer un seguimiento de los procedimientos empleados y los avances que han tenido con relación al objetivo de aprendizaje. El componente extensivo de las actividades favorece igualmente el replanteamiento de metas y la creación de nuevos objetivos tanto por los agentes educativos como por parte de los mismos educandos.

\section{Criterio 4: situaciones que favorezcan contextos complejos de interacción}

Un espacio educativo es significativo cuando dinamiza contextos de interacción complejos que favorecen la relación activa de los niños con el mundo. Un contexto de interacción rico y complejo es aquel que genera un mayor número de posibilidades de intercambio entre los aprendices y los objetos, los pares, los agentes educativos, los artefactos culturales y los eventos relevantes de todos los días. Tal intercambio tiene una función transformadora. Por el contrario, un contexto de interacción es pobre cuando los niños no tienen posibilidades de establecer relaciones transformadoras con su entorno.

Las prácticas cotidianas y culturales son contextos educativos pertinentes para implementar este criterio dado que involucran a los niños en variadas interacciones con otros compańeritos y adultos, con los instrumentos, eventos y metas de la comunidad, introduciéndolos en roles que les ofrecen un lugar central en el grupo cultural. Sin embargo, no basta con usar prácticas culturales para establecer un contexto de interacción complejo. En el ambiente de aprendizaje, la calidad de las modalidades de intervención de los agentes educativos es la que determina la calidad de las formas de interacción y participación de los aprendices, instaurando una dinámica entre los niños y la situación que puede favorecer o no, el logro de las metas educativas. Algunas de estas modalidades 
de intervención y formas de interacción son revisadas a continuación en el uso de prácticas culturales:

Resolución colaborativa de problemas. Una situación de resolución de problemas colaborativa es aquella que plantea un problema a un grupo de personas cuya respuesta sólo puede ser alcanzada por la acción y la interacción de todas las personas del grupo. Un problema que puede ser resuelto fácilmente por uno sólo de los integrantes no es una situación colaborativa. Coll (1997) señala que la resolución de problemas colaborativa es la forma de organización social que tiene mayor efecto en el aprendizaje de los sujetos que otras formas de organización social como la competencia o la resolución individual de tareas y problemas. Su importancia radica en que la comprensión grupal del problema así como la planeación y generación de estrategias conjuntas exige de la reflexión sobre el conocimiento de cada una de las personas del grupo y la negociación de este conocimiento entre ellos. Además exige la comunicación o socialización de los resultados de cada submeta y la meta en general. La situación Diseño de la granja comunitaria construida por los 3 agentes educativos de la escuela rural del Cauca para trabajar competencias ciudadanas, científicas, matemáticas y comunicativas (ver criterio 2), plantea varias situaciones de resolución de problemas que son resueltos de manera colaborativa entre los grupos de nińos y todos los grupos para alcanzar la meta de la actividad. Por ejemplo, las mediciones y la distribución de todo el terreno son logradas a partir de la medición y distribución de las eras de los grupos de niños, y a su vez éstas son medidas y distribuidas entre todos los miembros de un grupo. Para realizar el diario de registro con el plano y la sistematización de los datos, los niños deben socializar los resultados de su propia medición con los otros, y si uno se equivoca afecta la medición de todo el terreno. El agente educativo del Valle que trabaja la ronda "Agua de limón" para promover competencias sociales y matemáticas (ver criterio 1), también propone como meta de la tarea hacer grupos de tres o de cinco niños, que sin el trabajo colaborativo no puede ser alcanzada. En esta medida, los resultados de toda la actividad dependen de las acciones y las interacciones de los participantes.

Enseñar a otros niños las prácticas culturales. Algunos agentes educativos tienen la disposición permanente a presentar a los niños los juegos que utilizan, a dar las instrucciones y las reglas o a enseńar ellos mismos los estribillos y canciones. Con estas modalidades de intervención se genera una dinámica en la que los agentes educativos son directivos mientras los niños adoptan un papel pasivo, pues al jugar sólo siguen instrucciones y decisiones del adulto. Esta modalidad de intervención genera una forma de interacción unidireccional. Por el contrario, el agente educativo que utiliza la práctica "Agua de limón", al iniciar la activi- 
dad pregunta a los niños si alguna vez han jugado esta ronda y a quienes ya la conocen les pide que expliquen a los demás niños los pasos, las instrucciones y las reglas a seguir. Después promueve acuerdos entre ellos sobre algunas reglas de convivencia que se deberían cumplir. Además, propone que todos los niños jueguen -independientemente de la edad- y cuando ve que los más pequeñitos de 2 años se quedan a un lado sin cantar o bailar los invita a jugar. Para que lo hagan, pide a los niños de mayor edad que los tomen de la mano durante el juego y los ayuden a girar. Estas propuestas promueven un papel más activo, autónomo y responsable de los niños en la actividad y formas de interacción no sólo del agente educativo hacia los educandos, sino entre los mismos educandos.

La elección de roles por parte de los niños. En juegos tradicionales colombianos que involucran roles como "El gato y el ratón" o "El gavilán pollero", algunos agentes educativos elijen los niños que asumirán tales roles. Generalmente, proponen a los más grandes que se saben las canciones o los estribillos de memoria. Esta modalidad de intervención no permite que los niños tomen decisiones por sí mismos. Cuando los agentes educativos piden a los participantes que propongan quiénes quieren hacer de gato y de ratón, o de gavilán pollero y mamá gallina, $\mathrm{y}$ que entre todos elijan quiénes de los niños postulados van a asumir estos roles, les ofrecen una oportunidad para tomar decisiones de manera autónoma, negociada y basada en la evaluación de sus propias posibilidades. La elección de roles por parte de los niños en un juego constituye una modalidad de intervención favorable para generar una dinámica en la que desempeñan un papel más activo que los compromete con la actividad a realizar y a su vez promueve competencias ciudadanas como la participación social y la toma de decisiones.

Implementación de preguntas para enriquecer la situación. Un agente educativo puede únicamente llevar a cabo los pasos de un juego, bailar una ronda, cantar una canción de animales y al terminarlos detiene la actividad. Aunque los niños participan en los juegos, rondas y canciones, es posible que no haya una interacción rica entre ellos o con el conocimiento implicado en la práctica. Otros agentes educativos por el contrario, pueden plantear a los nińos preguntas durante o al final las prácticas con el fin de enriquecerlas. Por ejemplo, en la ronda "Agua de Limón", el agente educativo formula una serie de preguntas aditivas y multiplicativas al final del juego y así enriquece la práctica tradicional con el objetivo de generar en los niños significados más abstractos de números. Así propone problemas que pueden ser resueltos por cada grupo de participantes. En la práctica Diseño de la huerta comunitaria, las maestras formulan preguntas a los niños en la actividad de planificación para conocer sus ideas previas sobre una huerta y sobre sus beneficios para la comunidad, para generar una definición 
conjunta y negociada de "huerta comunitaria" y para construir entre todos, los pasos de las siguientes actividades. Durante la tercera actividad, de distribución, las maestras generan preguntas dirigidas a sistematizar la información que los niños obtienen en sus grupos, y a socializarla a los otros grupos. Las preguntas pueden llevar a los niños a comprender mejor las actividades y reflexionar sobre cómo participaron en ellas y cómo enfrentaron en equipo una meta común. Además, estas modalidades de intervención favorecen formas de interacción que van más allá de la relación agente educativo-educandos, promoviendo la relación educando-educando, educandos-artefactos culturales y educandos-metas culturales, entre otras posibles.

Consignas o instrucciones que permitan actuar sobre la realidad. En muchos de los ambientes educativos las actividades están centradas en la mente del agente educativo más que en la mente de los niños, por ejemplo, algunos maestros hacen la exposición de un tema o un dictado, dan órdenes o instrucciones que supuestamente permitirán el aprendizaje de los niños, o hacen afirmaciones sobre el conocimiento y exigen a los alumnos aprender de memoria estas "respuestas correctas". Tal dinámica está centrada en el reconocimiento del agente educativo como poseedor del conocimiento y del educando como el que debe únicamente recibir el conocimiento del agente educativo. Sin embargo, la dinámica del ambiente de aprendizaje cambia cuando los niños pueden formular propuestas o sugerencias, dar ejemplos o participar en la planificación y evaluación de una actividad. La dinámica igualmente cambia cuando se pide a los niños debatir con justificaciones y argumentos basados en sus conocimientos previos y en sus creencias, más que dar una respuesta correcta memorizada. Si los agentes educativos introducen frecuentemente preguntas y demandan propuestas, justificaciones, explicaciones, ejemplificaciones y argumentos a los niños, ellos empiezan a relacionarse con actividades fundamentales para la construcción de nuevo conocimiento como razonar, formular hipótesis, pensar, justificar y explicar sus puntos de vista. De esta manera, el agente educativo logra ir más allá de la mera transmisión de información y sugiere una participación más activa de sus niños.

Utilizar actividades de competencia. El uso de prácticas tradicionales como los juegos de competencia constituyen un escenario privilegiado para el desarrollo de competencias ciudadanas (Orozco, et al. 2001). Actividades como "El gato y el ratón", "El baúl de mi abuelita", "El Agua de Limón" y el "Gavilán Pollero", son prácticas tradicionales en algunos países de Latinoamérica, trabajadas por madres comunitarias del Valle-Colombia para promover la capacidad de pensar desde la perspectiva del otro, de elegir y tomar decisiones, de seguir reglas, de generar estrategias para ganar, de asumir consecuencias, de argumentar sus pro- 
pias ideas, de comprender y respetar las ideas, las creencias y los sentimientos de los demás, la cooperación, el trabajo en equipo y la solidaridad (Otálora, et al. 2007). Estas capacidades son necesarias para el desarrollo de habilidades sociales, de convivencia, de buena ciudadanía y la participación en su grupo cultural. Las competencias ciudadanas garantizan la participación de los niños como seres humanos responsables, respetuosos y capaces de convivir con otras personas.

Uso de instrumentos de la cultura. El uso de instrumentos creados o vinculados a las comunidades a las que los niños pertenecen promueve su interacción con el contexto cultural. Para Bruner (1997) un instrumento de la cultura funciona como un amplificador cognitivo que media entre las interacciones de sus miembros y los objetos de la acción. Así, el uso de canciones, nanas, danzas, mitos y cuentos propios de las regiones, dibujos, mapas y representaciones gráficas, juguetes tradicionales, instrumentos musicales, hojas, piedras y semillas, revistas, libros o periódicos locales son herramientas que además de facilitar la construcción de conocimiento de los nińos en dominios específicos, como sociales, matemáticas, físicas y el lenguaje, apoyan la comprensión de su propia historia y generan valiosas formas de interacción con los objetos, las tradiciones y las metas de la cultura, dando un sentido único a las actividades. El uso de nuevas tecnologías por otra parte, aunque traspasa los límites de las comunidades culturales, es útil para apoyar la construcción de conocimiento sobre otras culturas o la propia cultura en el contexto global.

Las modalidades de intervención revisadas son sólo algunas de las tantas que pueden llegar a generar formas de participación activa y legítima en la actividad y formas de interacción transformadora sobre el entorno. Las prácticas culturales por su parte pueden constituirse como contextos ricos y complejos de interacción que permiten a los niños definir rasgos de su personalidad, construir su identidad cultural, apropiarse de saberes, creencias y valores compartidos por una comunidad y fomentar el sentido de pertenencia a esta. A su vez, la interacción de los nińos con los objetos, los eventos cotidianos, con las formas de mediación y negociación que el otro utiliza o con los instrumentos y las metas de la cultura permite la construcción y transformación de procedimientos cada vez más complejos basados en la experiencia y favorecen el desarrollo de formas más avanzadas de pensar y de relacionarse con otros, que a su vez permiten a los nińos enfrentarse a problemas de mayor complejidad en la sociedad. Estos contextos permiten que los nińos participen como interlocutores válidos, compartiendo sus propias creencias, concepciones y conocimientos. 


\section{Criterio 5: situaciones generativas que exijan múltiples competencias}

Ejercicios estereotipados como hacer planas en un papel o bolitas de plastilina, resultan de poco interés para los niños porque repiten un sinnúmero de veces la misma actividad sin que tenga un sentido para ellos. Escribir incansablemente el número "dos" no quiere decir que los niños comprendan cuánto es "dos" o que sean capaces de sumar y restar con el "dos". Los niños únicamente están trabajando su motricidad fina. Estas son situaciones no generativas porque los niños sólo desarrollan una competencia, pero además, porque esta competencia no implica pensar. Tales actividades resultan poco significativas porque los niños no tienen que utilizar conocimientos y capacidades variadas para cumplir con la tarea y de esta manera no les facilita comprender el mundo ni aprender a pensar mejor. Un espacio educativo es significativo cuando es generativo, es decir, cuando su demanda exige "generar redes amplias de relaciones, dentro de un dominio de conocimiento y entre dominios de conocimiento". En otras palabras, cuando permite a los niños el uso de múltiples competencias.

La ronda "Agua de limón" (ver criterio 1) permite ilustrar la manera como en una secuencia de actividades un agente educativo es capaz de fortalecer simultáneamente competencias matemáticas y ciudadanas. Para dar respuesta a las preguntas de cuantificación, cardinalidad, suma y multiplicación, los niños deben comprender y seguir reglas, trabajar en equipo y además, los chicos grandes deben hacerse responsables de los más pequeños. Incluso con una sola ronda la madre comunitaria logra construir un ambiente de aprendizaje que permite generar múltiples relaciones en un mismo dominio de conocimiento, como por ejemplo, relaciones aditivas y multiplicativas, y establecimiento de cardinales o totales abstractos en la competencia matemática y el manejo de reglas, la toma de decisiones, el trabajo en equipo y la solidaridad en la competencia social. La actividad Diseño de una huerta comunitaria (ver criterio 2) permite trabajar la toma de decisiones, el trabajo en equipo y el manejo de reglas en la competencia social, la formulación de hipótesis y la planificación en la competencia científica, el establecimiento de relaciones espaciales y geométricas, la cuantificación y la comprensión de los usos y las formas de medición, en la competencia matemática y la comprensión y producción de textos, la argumentación, la socialización de ideas y resultados y la negociación de significados en la competencia comunicativa.

Para que una situación pueda exigir competencias variadas es más efectivo plantear una temática central suficientemente amplia y compleja, también llamado tópico generativo (Stone, 1997), que permita articular un conjunto de actividades dirigidas a metas de aprendizaje o comprensión específicas. En la 
actividad Diseño de la huerta comunitaria las maestras eligieron como temática central construir conjuntamente con los nińos una huerta donde sembraran frijol, maíz y cilantro que resulta de gran beneficio para los miembros de la comunidad rural en la que se encuentra la escuela. Esta temática es lo suficientemente amplia y compleja como para trabajar una gran variedad de conocimientos y saberes con niños de diferentes edades, a lo largo de todo el semestre. Alrededor de una temática central es posible recrear diferentes contextos significativos que le dan sentido a la situación. La presencia de la temática central y los contextos significativos posibilita la organización de una situación que favorece la comprensión de los niños, la generación de una vasta red de relaciones conceptuales y la construcción ó el descubrimiento de nuevas herramientas del pensamiento.

Durante esta segunda parte del artículo se ha ilustrado la manera como el uso de prácticas culturales permite la operacionalización de los cinco criterios propuestos para que un espacio educativo pueda constituirse como significativo para el desarrollo de competencias en la primera infancia. Esta ilustración ha buscado igualmente mostrar cómo se pueden tener en cuenta las implicaciones de la concepción de desarrollo infantil y la definición del ambiente de aprendizaje como un escenario de construcción de conocimiento dinámico y complejo en el diseño o enriquecimiento de los espacios educativos.

\section{Bibliografía}

Brown, John, Collins, Allan y Duguid, Paul (1989). "Situated cognition and the culture of learning". Educational Researcher, 18, 1, 32-41.

Bruner, Jerome (1997). "Cultura, mente y educación". La Educación, Puerta de la Cultura. Aprendizaje Visor, Madrid.

Coll, César (1997). Estructura grupal, interacción entre alumnos y aprendizaje escolar. Aprendizaje escolar y construcción de conocimiento, Capitulo 5, 105-130. Paidós, Barcelona.

Coll, César y Onrubia, Javier (1996). "La construcción de significados compartidos en el aula: actividad conjunta y dispositivos semióticos en el control y seguimiento mutuo entre profesor y alumnos". En: Coll, César y Edwards, Derek (eds.) Enseñanza, aprendizaje y discurso en el aula. Aproximaciones al estudio del discurso educacional, Capitulo 4, 53-73. Aprendizaje, S. L., Madrid. 
Gelman, Rochel (2000). "Domain specificity and variability in cognitive development". Child Development, 71, 4, 854-856.

Karmiloff-Smith, Annette (1996). "El desarrollo tomado en serio". Más allá de la modularidad. La ciencia cognitiva desde la perspectiva del desarrollo. Capitulo 1, 17-49. Alianza, Madrid.

Luria, Aleksander Romanovich y Tsvetkova, L.S. (1981). La resolución de problemas $y$ sus transtornos. Fontanella, Barcelona.

Orozco, Mariela; Ochoa, Solanlly y Sánchez, Hernán (2001). Prácticas culturales para la educación de la niñez. Fundación Antonio Restrepo Barco, Bogotá

Otálora, Yenny (2007). "Espacios educativos significativos para la educación en la infancia". En Yenny Otálora \& Marlenny Guevara (comps.) Recuperando y Significando Ando. Prácticas Culturales para la Comprensión y Promoción del Desarrollo Infantil. Artes Gráficas del Valle, Cali.

Otálora, Yenny (2009). Análisis de tareas como estrategia metodológica para acceder a la cognición encubierta. Centro de Investigaciones en Psicología, Cognición y Cultura, Cali.

Otálora, Yenny y Guevara, Marlenny (2007). Recuperando y Significando Ando. Prácticas Culturales para la Comprensión y Promoción del Desarrollo Infantil. Artes Gráficas del Valle, Cali.

Puche-Navarro, Rebeca (2003). El niño que piensa y vuelve a pensar. Artes Gráficas del Valle, Cali.

Newell, Allen y Simon, Herbert (1972). Human Problem Solving. Englewood Cliffs, Prentice-Hall, New Jersey.

Siegler, Robert (2007). "Cognitive variability". Developmental Science, 10, 1, 104-109.

Steffe, Leslie (1990). "Cómo construye el niño la significación de los términos aritméticos". Cuadernos de Psicología, 11, 1, 107-162.

Stone, Martha (1999). "¿Qué es la enseñanza para la comprensión?". En La enseñanza para la comprensión, Capitulo 3, 95-126. Paidós, Buenos Aires. 